\title{
Avaliação do estado nutricional da comunidade indígena Kaingang. Área Monte Caseiros - Muliterno RS
}

\author{
Evaluation of the nutricional status of the Kaingang indigenou \\ community. Monte Caseiros Area - Muliterno RS
}

Jeanine Eggers Caramori ${ }^{1}$

Ernesto Grazziotin Longhi ${ }^{2}$

\section{Resumo}

A obesidade tornou-se um problema mundial, no qual a dieta alimentar não-balanceada contribui, e muito, para o aumento de peso. Realizou-se um estudo com o objetivo de conhecer a prevalência da obesidade e dos fatores de risco que levam ao desenvolvimento dessa patologia, dando ênfase a alguns fatores de risco e o perfil alimentar da comunidade indígena da Estratégia da Saúde da Família da Aldeia Monte Caseiros de Muliterno (RS). Foram entrevistados, por meio de questionários, medidos e pesados 143 indivíduos, maiores de 20 anos e registrados no Programa SIASI 2008. Foram avaliados pelo índice de massa corporal (IMC), e os resultados foram: 40\% de obesos, 38\% de pessoas com sobrepeso e apenas $22 \%$ com o peso adequado. No grau de obesidade, $60 \%$ são mulheres, $80 \%$ realizam três refeições diárias, 95\% preparam a refeição com banha de porco e 49\% não realizam nenhuma atividade física. De modo semelhante a outros estudos com populações distintas, conclui-se que, entre os Kaingangs, o sobrepeso e a obesidade acometem um grande número de indivíduos.

\begin{abstract}
The obesity became a world wide health problem, which a non-balanced diet contributes significantly for the weight increasement. A study was made to know the obesity prevalence and risk factors that contribute with the development of this patology, emphasizing some risk factors and the alimentary profile of Indigena Community for Family Health Strategy of Monte Caseiros Village from Muliterno, RS, Brazil. Interviews were made, within questionnaire, tallness and weight measurement (143 individuals, 20 years old higher aged, registered on SLASI Program - 2008. Body Mass Index was considered and the result was that: $40 \%$ individuals with obesity, 38\% overweight and just $22 \%$ on suitable weight. In obesity level, $60 \%$ are women, $80 \%$ bave 3 daily meals, 95\% prepare their meals with pig grease and $49 \%$ don't exercise. Comparing other studies with other people, different than this one, we can conclude that among the Kaingangs, overweight and the obesity affect a big number of individuals.
\end{abstract}

Palavras-chave: Antropometria; Avaliação Nutricional; Obesidade; Índios Sul-Americanos; Sobrepeso; Índice de Massa Corporal; Saúde Indígena.
Key Words: Anthropometry; Nutrition Assessment; Obesity; Indians; South American;

Overweight; Body Mass Index; Indigenous Health.

${ }^{1}$ Médica de Familia e Comunidade, Estrategia da Saude da Familia - Area Indigena Monte Caseiros, Muliterno, Rio Grande do Sul, Brasil.

${ }^{2}$ Academico do $9^{\circ}$ semestre da Faculdade de Fisioterapia da Universidade de Passo Fundo, Passo Fundo, Rio Grande do Sul, Brasil. 


\section{Introdução}

A obesidade, no século atual, é um problema de origem universal; é uma doença crônica decorrente da interação entre vários fatores: genéticos, ambientais, sociais, econômicos, culturais, nutricionais, metabólicos, psicológicos, neurológicos e endócrinos ${ }^{1,2}$. Ela está associada ao surgimento de enfermidades crônicas, como diabetes mellitus tipo II, dislipidemias ${ }^{3}$, hipertensão arterial ${ }^{4,5}$, doenças cardiovasculares $^{6}$ e diversos tipos de câncer ${ }^{7}$.

A Organização Mundial da Saúde ${ }^{8}$ registrou um aumento significativo da obesidade nas últimas décadas, sendo esta considerada epidemia mundial, independentemente das condições econômicas e sociais, atingindo tanto países desenvolvidos como aqueles em desenvolvimento. O diagnóstico analítico da situação alimentar e nutricional tem contribuído para que se conheça a natureza e a magnitude dos problemas de nutrição em determinada área indíigena e a avaliação do estado nutricional de indivíduos para obter o diagnóstico precoce dos possíveis desvios nutricionais, evitando as consequências decorrentes desses agravos à saúde.

\section{Critérios para o diagnóstico e o acompanhamento do estado nutricional}

O estado nutricional é o resultado do equilíbrio entre o consumo de nutrientes e o gasto energético do organismo para suprir as necessidades nutricionais. Este pode ter três tipos de manifestações orgânicas:

- Adequação Nutricional (Eutrofia) - manifestação produzida pelo equilíbrio entre o consumo em relação às necessidades nutricionais.

- Carência Nutricional: manifestação produzida pela insuficiência quantitativa e/ou qualitativa do consumo de nutrientes em relação às necessidades nutricionais.

- Distúrbio Nutricional: manifestação produzida pelo excesso e/ou desequilíbrio de consumo de nutrientes em relação às necessidades nutricionais.

O método de escolha preconizado a ser usado na avaliação nutricional para a vigilância do estado nutricional (SISVAN) é o antropométrico. A antropometria é um mé- todo de investigação em nutrição com base na medição das variações físicas e na composição corporal global. É aplicável em todas as fases da vida e permite a classificação de indivíduos e grupos segundo o seu estado nutricional, tendo como vantagem ser barato, simples, de fácil aplicação e padronização, além de pouco invasivo.

Além de tudo, possibilita que os diagnósticos individuais sejam agrupados e analisados de modo a fornecer o diagnóstico coletivo, permitindo conhecer o perfil nutricional de um determinado grupo. É universalmente aceita e apontada como o melhor parâmetro para avaliar o estado nutricional de grupos populacionais. Os dados antropométricos são peso e altura. Com esses dados, podese calcular os índices antropométricos ou nutricionais mais utilizados, lembrando que cada uma das fases do ciclo de vida possui referências e pontos de corte diferenciados.

\section{Pontos de corte estabelecidos para adultos}

IMC

$$
\begin{array}{ll}
<18,5 \ldots 18,5 \mathrm{e}<25 \ldots & \text { baixo peso } \\
& \text { adequado } \\
& \text { (eutrófico) } \\
>25<30 & \text { sobrepeso } \\
& \text { obesidade }
\end{array}
$$

Os limites de normalidade são chamados de ponto de corte. Pela classificação do SISVAN (para os ciclos de vida), os adultos são maiores ou iguais a 20 e menores que 60 anos de idade.

Nos procedimentos de diagnóstico nutricional de adultos, a Vigilância Alimentar e Nutricional (SISVAN) utiliza a classificação do Índice de Massa Corporal (IMC), recomendada pela Organização Mundial da Saúde - OMS .

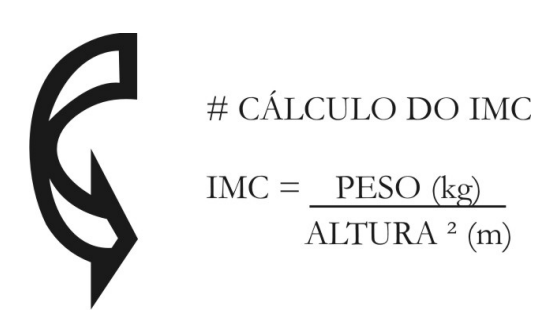




\section{Materiais e Métodos}

Foi aplicado questionário com perguntas objetivas para cada indígena da Aldeia Monte Caseiros (Kaingangs) - todos os envolvidos (143) maiores de 20 anos e cadastrados no Programa SIASI - 2008, durante o mês de novembro do ano de 2008 - sobre estilo de vida e alimentação. Foram realizadas pesagem e medida da altura em uma balança mecânica do tipo plataforma (Balmak n ${ }^{\circ} 10481$ $\mathrm{IN}=2.642 .649-3$ ) com capacidade para $150 \mathrm{~kg}$ (precisão de $50 \mathrm{~g}$ ) e com extensão de 2,13 m (precisão de $1 \mathrm{~mm}$ ).

Depois de coletadas todas as medidas, foram utilizadas as seguintes referências para avaliar o estado nutricional, conforme recomendações do Ministério da Saude e da Organização Mundial da Saude (SISVAN/ 2004). Após a coleta dos dados, estes foram digitados e analisados, usando-se os programas Excel 2007 e SPSS 17.0.

\section{Resultados}

Conforme a avaliação dos dados coletados utilizando-se o Índice de Massa Corporal - o ponto de corte preconizado (SISVAN - 2004) -, obtivemos entre os indivíduos pesquisados: $40 \%$ de obesos, $38 \%$ de pessoas com sobrepeso e apenas $22 \%$ com o peso adequado; não foi encontrado nenhum em risco nutricional $(p<0,01)$. (Gráfico 1)

Avaliando-se sobre o ponto de vista do grau de obesidade relacionado ao sexo dos indivíduos, as mulheres são a maioria $(60 \%)$ contra $40 \%$ de homens. (Gráfico 2)

Em relação à refeição, somente $20 \%$ não realizam as três principais refeições diárias. (Grafico 3) O tipo de gordura utilizada na alimentação influencia bastante, pela quantidade de gorduras saturadas que possui - 95\% dos indivíduos preparam as refeições com banha de porco, o que reflete no grau de obesidade da população. (Gráfico 4)

Observou-se que a porcentagem de indígenas que realizam atividade física (uma ou mais vezes por semana) foi de $51 \%$, sendo que $49 \%$ não realizam nenhum tipo de atividade física. (Gráfico 5)
Gráfico 1. Representa a prevalência dos indivíduos, com seus índices de massa corporal, conforme a classificação nutricional preconizada pelo SISVAN - 2004.

\section{Índice de Massa Corporal}

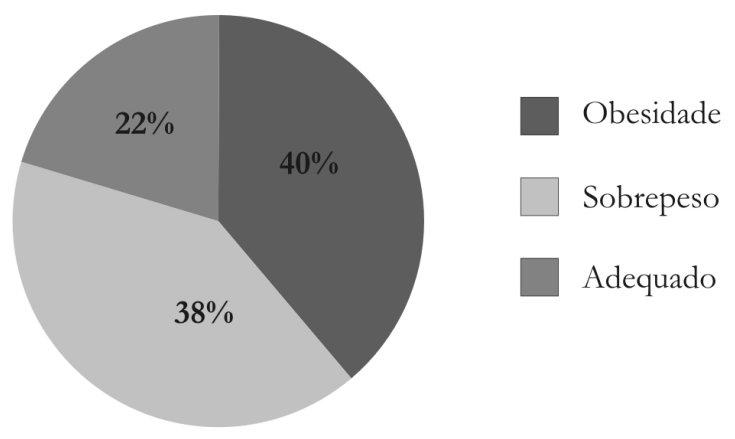

Gráfico 2. Representa a prevalência de obesidade relacionada ao sexo.

\section{Obesidade}

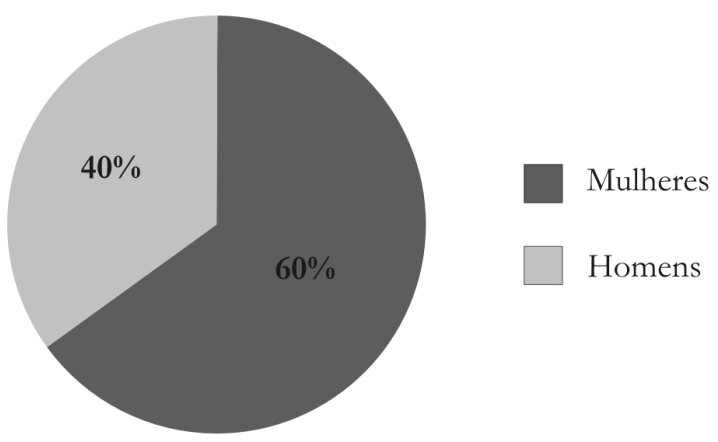

Gráfico 3. Representa a prevalência dos indivíduos quanto ao tipo de gordura que utilizam para o preparo das refeições.

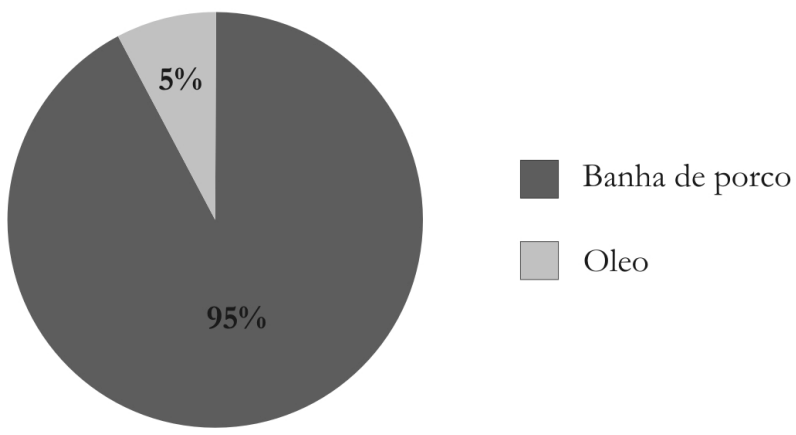


Gráfico 4. Representa a prevalência de indivíduos em relação às três refeições diárias.

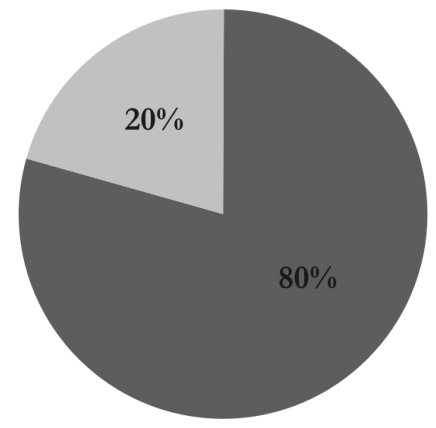

Realizam 3 refeições diárias

Não realizam 3 refeições diárias

Gráfico 5. Representa a prevalência de indivíduos sobre a realização de atividade física.

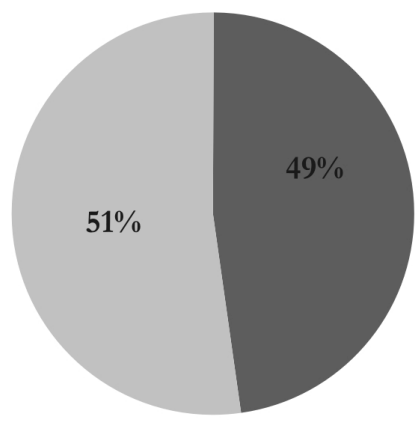

Realizam atividade física

Não realizam atividade física

\section{Discussão}

No Brasil, dados apresentados pela Pesquisa Nacional de Saúde mostram que, entre 1974 e 1989, a proporção de pessoas com excesso de peso aumentou de $21 \%$ para 32\%. Entre as regiões do país, as maiores prevalências são registradas na Região Sul, sendo estas semelhantes, ou até mesmo superiores, àquelas descritas em países desenvolvidos. Os dados apresentados mostram, ainda, que o sobrepeso e a obesidade elevam-se com a idade, alcançando maior prevalência na faixa etária de 45 a 54 anos, na qual foi registrado aumento do peso corporal acima dos limites preestabelecidos em 37\% dos homens e 55\% das mulheres $^{10}$.

Alterações do peso em níveis que ultrapassam o limite superior de normalidade estabelecido em 1997 pela
Organização Mundial da Saúde (OMS) constituem, no mundo atual, um dos mais sérios e frequentes problemas de saúde.

A OMS registrou, em, 2002 a existência de mais de um bilhão de adultos com excesso de peso, sendo 300 milhões considerados obesos ${ }^{11}$. No Brasil, a prevalência de obesidade em adultos com 20 anos ou mais no período de 1975 a 1989 quase dobrou, passando de 4,4\% para $8,2 \%$, alcançando $9,7 \%$ em 1997 . A prevalência de sobrepeso passou, no mesmo período, de $21 \%$ para $32 \%{ }^{12}$.

A grande maioria dos estudos revela uma maior prevalência de sobrepeso e obesidade entre as mulheres, conforme o resultado deste estudo.

O presente trabalho procurou avaliar a prevalência de obesidade na comunidade indígena Kaingang. Os resultados encontrados mostram $40 \%$ de obesidade na população e $38 \%$ de sobrepeso, com base na tabela de ponto de corte do IMC pelo SISVAN - 2004. Por outro lado, Capelli et al. encontraram uma prevalência maior de sobrepeso na população adulta em seu estudo com indígenas Parkatejề $\hat{~}^{13}$.

Este estudo documentou a associação com o sexo, sendo a prevalência maior (60\%) no sexo feminino e com estilo de vida. Assim, observa-se que, quanto a esse aspecto, Capelli et al. também observaram uma prevalência de $41,3 \%$ de obesidade na população feminina e $24,6 \%$ na masculina.

Verificou-se que a maioria dos indivíduos utiliza banha de porco para preparar as refeições $(95 \%)$ e realiza três refeições diárias (80\%) e que $49 \%$ dos estudados não realizam atividade física, fatos que contribuem muito para o aumento de peso.

A discussão deve estender-se além da simples análise de dados de prevalência. Há de se conhecer a etiopatogenia e fisiopatologia da obesidade, juntamente com as condições sociais nas quais vivem estes indivíduos como: falta de saneamento ou saneamento inadequado, condições precárias de moradia, situação fundiária limitada, infraestrutura local, esgotamento da produtividade de terra, alimentação precária, dentre outras. 
Em termos alimentares, os Kaingangs eram tradicionalmente caçadores/coletores, com agricultura e pesca incipientes ${ }^{14}$.

Antes do contato, ocorrido em décadas passadas, os Kaingangs possuíam uma alimentação balanceada, a partir da caça e da coleta de alimentos. Hoje, com a sedentarização pela invasão cultural ocasionada pela comunidade nas reservas indígenas Kaingangs, restou pouco das suas origens alimentares. O consumo de amido em excesso, aliado ao uso de açúcar, hábito obtido após o contato, propicia o aparecimento de obesidade além de outras doenças nas comunidades indígenas ${ }^{14}$.

\section{Conclusão}

Semelhante ao que vem sendo apontado em estudos no país e em pesquisas com outras populações indígenas, observou-se que, entre os Kaingangs, o sobrepeso e a obesidade acometem grande número de indivíduos.

Estudos nacionais apontam que o aumento do sobrepeso e da obesidade na população pode ser causado pela redução da atividade física e pela modificação alimentar. Estes estudos apontam que o índio brasileiro modificou sua alimentação nas últimas décadas, consumindo maior quantidade de alimentos calóricos, refinados, ricos em açúcar e sal e pouca quantidade de alimentos mais naturais e ricos em fibras, como frutas, legumes e verduras.

\section{Referências}

1. Carvalho KMB. Obesidade. In: Cuppai L. Guias de Medicina Ambulatorial e Hospitalar. UNIFESP/Escola Paulista de Medicina. Nutrição: nutrição clinica no Adulto. São Paulo: Manole; 2002. p. 131-65.

2. Araujo LMB. Obesidade. In: Rabelo MM. Manual pratico de endocrinologia. São Paulo: Fundo Editorial BYK; 2003. p. 362-65.

3. Manson JE et al. A prospective study of obesity and risk of coronary heart disease in women. N. Engl. J. Med. 1990; 322: 882-89.

4. Bouchard C, Depres JP, Tremblay A. Exercise and obesity. Obesity Res. 1993; 1: 133-47.
5. Ryan AS et al. Relationship of blood pressure to fatness and fat patterning in Mexican American adults from the Hispanic health and nutrition examination survey (NHANES, 1982 - 1984). Coll. Antropol. 1994; 18: 89-99.

6. Mykkanen L, Laakso M, Pyorala K. Association of obesity and distribution of obesity with glucose tolerace, and cardiovascular risk factors in the eldery. Int. J. Obesity. 1992; 16: 695-704.

7. Garfinkell L. Overweight and cancer. Ann. Intern. Med. 1985; 103: 1034-36.

8. World Health Organization. Obesity prevening and managing. The Global Epidemic. Report of a Who Consultation on Obesity. Geneva: WHO; 1998.

9.Vigilancia Alimentar e Nutricional-SISVAN: Orientações basicas para a coleta, processamento, analise de dados e informação em serviços de saude. Brasília (DF): Ministerio da Saude, 2004.

10. Caires NFR et al. Sobrepeso e Obesidade entre os funcionários da UEFS. Rev. Baiana de Saude Publica. 2005; 29(2): 238-50.

11. World Health Organization. Obesity and overweight. Disponível em: http://www.who.int/dietphysicalactivity/ publications/facts/obesity/em/. Acessado em: nov. 2008. 12. Monteiro CA, Conde WL, Popkin BM. Independent effects of income and education on the risk of obesity in the Brazilian adult population. J. Nutrion. 2001; 131(Supl.): 881S-886S.

13. Capelli et al. Avaliação do Estado Nutricional da Comunidade Indigena Parkatêjê, Bom Jesus da Tocantins, Pará, Brasil. Cad. Saude Publica. 2001; 17 (2): 433-37.

14. Freitas et al. Influencia da mudança do habito alimentar na prevalência de diabetes na área Indigena Xavante. Estudo de caso - Reserva Indígena São Marcos. Brasília (DF): Boletim de Pesquisa; 2004. 


\section{Endereço para correspondência:}

Av. Genuíno Antônio Astolfi, 607.

Muliterno - RS

CEP: 99990-000

\section{Endereço eletrônico:}

jecaramori@yahoo.com.br 